\title{
Abdominal pain associated with cranial nerve palsy and peripheral neuropathy
}

\author{
LK Dawson, CD Selby, KCH Fearon, CJ Mumford, ER Chilvers
}

University of Edinburgh, The Royal Infirmary, Edinburgh EH3 9YW, UK Department of Medicine LK Dawson CD Selby ER Chilvers Department of Surgery KCH Fearon

Department of Clinical Neurosciences, Western General Hospital, Crewe Road South, Edinburgh EH4 2XU, UK CJ Mumford

Correspondence to ER Chilvers

Accepted 28 February 1996
A 61-year-old woman was admitted with a four-week history of right-sided pleuritic chest pain. One day prior to admission the pain had moved to the epigastrium with radiation to the upper lumbar spine. The pain was constant and severe and associated with marked anorexia and nausea. There was no vomiting or bowel disturbance. Her medical history included tuberculosis at the age of three affecting her left hip. She subsequently underwent hip arthroplasty at the age of 18 and total joint replacement earlier in the year of admission. She had had reactivation of her pulmonary tuberculosis aged 29 and an upper gastrointestinal bleed in 1991. At the time of presentation she was smoking 15 cigarettes per day.

On examination she was pale and distressed with a pulse rate of 70 beats $/ \mathrm{min}$, blood pressure of $140 / 80 \mathrm{mmHg}$ and a respiratory rate of $16 \mathrm{breaths} / \mathrm{min}$. Auscultation of her chest revealed fine inspiratory crepitations up to the mid zones bilaterally. She was tender on palpation of her upper lumbar spine and had grade $4+/ 5$ weakness of hip flexion on the left side. Initial investigations showed no radiological evidence of intra-abdominal free gas or abnormality of her lumbar spine. The chest X-ray showed evidence of previous pulmonary tuberculosis only. Routine biochemical and haematological parameters were normal apart from a white blood cell count of $11 \times 10^{9} / 1$, and erythrocyte sedimentation rate of $44 \mathrm{~mm} / \mathrm{h}$. Her serum amylase was $217 \mathrm{U} / \mathrm{l}$.

Following admission she developed acute retention of urine and began to complain of increasingly severe upper abdominal pain and lower thoracic pain. Repeat examination revealed increased epigastric tenderness but no evidence of spinal cord compression. Surgical review raised the possibility of a pancreatic carcinoma, gallbladder disease or a posterior penetrating duodenal ulcer. However, abdominal ultrasound, computed tomography and upper gastrointestinal endoscopy were all normal. Her serum amylase remained persistently under $500 \mathrm{U} / 1$. Sputum and early morning urine samples showed no acid-alcohol fast bacilli. In view of the unremitting nature and severity of her abdominal pain, which was only partially relieved by large doses of intravenous diamorphine, diagnostic laparoscopy was considered. However, over the ensuing 12 hours she reported difficulty sitting upright, an intense feeling of cold in both legs and right-sided facial weakness. Examination showed a right lower motor neuron facial nerve palsy, profound truncal and lower limb weakness with diminished reflexes and reduced sensation in both legs. Upper limb examination was normal. Further investigations at this stage demonstrated a normal serum lead level and antineutrophil cytoplasmic antibody titre, negative syphilis serology and auto-antibody screen. Urinary porphyrins were not detected. Magnetic resonance imaging (MRI) of the entire neuraxis and isotope bone scan were also normal. Cerebrospinal fluid (CSF) examination showed a white cell count of less than $1 \mathrm{cell} / \mathrm{mm}^{3}, \mathrm{CSF}$ glucose of $3.9 \mathrm{mmol} / 1$ (paired plasma glucose $6.5 \mathrm{mmol} / \mathrm{l}$ ) and an elevated protein of $3.15 \mathrm{~g} / 1$ (normal range $0.15-0.45 \mathrm{~g} / \mathrm{l})$. No malignant cells were identified in the CSF.

\section{Questions}

1 What is the most likely diagnosis?

2 Name three conditions which are associated with this disorder.

3 What is the current management of this condition? 
Answers

\section{QUESTION 1}

Guillain-Barré syndrome (acute inflammatory demyelinating polyneuropathy). The differential diagnosis of a patient presenting with abdominal pain and neurological signs includes acute intermittent prophyria, lead poisoning and carcinomatous meningitis. These diagnoses are unlikely in this case due to the absence of associated physical signs or supportive laboratory investigations. Alternative causes of an acute polyneuropathy in the absence of abdominal pain would include Lyme borreliosis, chronic inflammatory demyelinating polyneuropathy, HIV infection, sarcoid neuropathy, diphtheritic neuropathy, collagen vascular diseases, poliomyelitis, neoplastic and paraneoplastic neuropathy and paraproteinaemic neuropathy.

\section{QUESTION 2}

In $50 \%$ of patients with Guillain-Barré syndrome an identifiable infection precedes the onset of the neuropathy. This is commonly an upper respiratory tract or gastrointestinal infection (box). More rarely, Guillain-Barré syndrome may complicate surgery, pregnancy, epidural anaesthesia or Hodgkin's lymphoma.

\section{OUESTION 3}

Most cases of Guillain-Barré syndrome recover spontaneously and hence supportive measures are usually sufficient. Corticosteroids have no beneficial effect. Recent research suggests that plasma exchange and infusion of intravenous immunoglobin given in the early stages of the condition may lessen the severity and speed recovery.

\section{Discussion}

The delay in making the diagnosis of Guillain-Barré syndrome (acute inflammatory demyelinating polyneuropathy) in this patient was due in part to the atypical presentation, with severe abdominal pain mimicking acute intra-abdominal pathology, and also the con-

1 Ropper AH. Severe acute Guillain-Barré syndrome. Neurology 1986; 36: 429-32.

2 Winer JB, Hughes RA, Osmond C. A prospective study of actue idiopathic neuropathy. I. Clinical features and their prognostic value. $₹$ Neurol Neurosurg Psychiatry 1988; 51 : $605-12$.
Infections associated with GuillainBarré syndrome

- cytomegalovirus

- Epstein-Barr virus

- Campylobacter jejuni

- HIV

- mycoplasma

- parvovirus B19

- Yersinia enterocolitica

- toxoplasma

- herpes zoster

- non-A, non-B hepatitis virus

siderable delay before the development of neurological signs. While sensory symptoms are not uncommon in the Guillain-Barré syndrome, cranial nerve palsies occur in about $50 \%$ of cases. ${ }^{1}$ It is less well recognised, however, that about half of all patients with the Guillain-Barré syndrome experience pain, although this is usually located in the legs, lower trunk or lumbar region. ${ }^{2,3}$ The pain is characteristically present at an early stage and precedes the development of neurological signs. In a study of 50 fatal cases, Haymaker and co-workers ${ }^{3}$ reported abdominal pain occurring in four patients which subsided as the disorder progressed, although in one case severe pain did persist and was associated with urinary retention. Autonomic disturbances causing wide variations in blood pressure and heart rate, paralytic ileus, bladder atony and facial flushing may also be observed and are associated with a poorer prognosis. The development or co-existence of neurological signs in patients with abdominal pain, especially if associated with back pain, should therefore raise the possibility of the Guillain-Barré syndrome.

\section{Final diagnosis}

Guillain-Barré syndrome

Keywords: abdominal pain, polyneuropathy, Guillain-Barré syndrome

\footnotetext{
3 Haymaker W, Kernohan JW. The Landry-Guillain-Barre
} syndrome. Medicine 1949; 28: 59-141. 